\title{
ADOMIAN DECOMPOSITION AND SUCCESSIVE APPROXIMATION METHODS FOR SOLVING KAUP-BOUSSINESQ SYSTEM
}

\author{
Saad A. Manaa ${ }^{\mathrm{a}^{*}}$, Nergiz M. Mosa ${ }^{\mathrm{b}}$ \\ a Department of Mathematics, Faculty of Science, University of Zakho, Kurdistan Region, Iraq - (saad.manaa@uoz.edu.krd) \\ ${ }^{\mathrm{b}}$ Department of Mathematics, College of Basic Education, University of Duhok, Kurdistan Region, Iraq \\ Received: May, 2019 / Accepted: July, 2019 / Published: Sep., 2019 \\ https://doi.org/10.25271/sjuoz.2019.7.3.582
}

\begin{abstract}
:
The Kaup-Boussinesq system has been solved numerically by using two methods, Successive approximation method (SAM) and Adomian decomposition method (ADM). Comparison between the two methods has been made and both can solve this kind of problems, also both methods are accurate and has faster convergence. The comparison showed that the Adomian decomposition method much more accurate than Successive approximation method.
\end{abstract}

KEYWORDS: Adomian Decomposition Method (ADM), Successive Approximation Method (SAM), Kaup-Boussinesq System (KB)

\section{INTRODUCTION}

The partial differential equations originated from the study of surfaces in geometry and for solving a wide variety of problems in mechanics. During the second half of the nineteenth century, a large number of mathematicians became actively involved in the investigation of numerous problems presented by partial differential equations (Debnath, 2011). Many authors have worked on partial differential equations such as: (Hosseini, Ansari \&Gholamin, 2012; Koparan et al., 2017).

An American mathematician has initiated and developed the Adomian decomposition method (Adomian, 1986, 1994). Moreover, this method is very helpful for a number of areas such as: for solving ordinary and partial equations, linear and nonlinear, Algebraic equations, factional equations and integral differential equations (awawdah, 2016). Also, it has been applied to a wide class of deterministic and stochastic problems, linear and nonlinear, in physics, biology and chemical reactions etc. For nonlinear models, the method has shown reliable results in supplying analytical approximations that converge rapidly(Chen $\& \mathrm{Lu}, 2004)$.The main advantages of the method are that it can be applied directly for all types of differential and integral equations, linear or nonlinear, homogenous or inhomogeneous, with constant coefficients or with variable coefficients. Another Advantages is that the method is capable of greatly reducing the size of computation work while still maintain high accuracy of the numerical solution (Somali \& Gokmen, 2007).

(Biazar \& Ghazvini, 2009) State that one of the classical methods for finding the solution of integral equations is the Successive approximation method (SAM). Moreover, in the literature is called Picard iteration method. Scheme that one can use for solving initial value problems (Adam, 2015; Biazar \& Ghazvini, 2009). Many authors such as (Adam, 2015; Hashem, 2015; Javadi, 2014; Manaa, Easif, \& Ali, 2017 ) have consideration to study linear and nonlinear PDEs by using SAM. In this work, $\mathrm{ADM}$ and SAM have been applied to solve the Kaup-Boussinesq system.

A coupled system of nonlinear partial differential equation which derives as a sample for surface waves in the context of Boussinesq scaling is the Kaup-Boussinesq system, and also been

\footnotetext{
* Corresponding author
}

This is an open access under a CC BY-NC-SA 4.0 license (https://creativecommons.org/licenses/by-nc-sa/4.0/) derived for an internal wave system (Juliussen, 2014; Zhou, Tian \& Fan, 2009).

The KB-system (Zhou, Tian \& Fan, 2009) :

$$
\left.\begin{array}{rl}
\mathrm{u}_{\mathrm{t}}-\mathrm{v}_{\mathrm{xxx}}-2(\mathrm{vu})_{\mathrm{x}} & =0 \\
\mathrm{v}_{\mathrm{t}}-\mathrm{u}_{\mathrm{x}}-2 \mathrm{v} \mathrm{v}_{\mathrm{x}} & =0
\end{array}\right\}
$$

With the initial conditions:

$$
\begin{aligned}
\mathrm{u}(\mathrm{x}, 0)= & \frac{\mathrm{w}^{2}}{2}\left(1+\tanh \left(\frac{\mathrm{wx}}{2}\right)\right) \\
& -\frac{\mathrm{w}^{2}}{4}\left(1+\tanh \left(\frac{\mathrm{wx}}{2}\right)\right)^{2}, \\
\mathrm{v}(\mathrm{x}, 0)= & -\frac{\mathrm{w}}{2}\left(1+\tanh \left(\frac{\mathrm{wx}}{2}\right)\right) .
\end{aligned}
$$

Where $u=u(x, t)$ indicate to the height of the water surface above a horizontal bottom, $\mathrm{v}=\mathrm{v}(\mathrm{x}, \mathrm{t})$ is related to the horizontal velocity field and $\mathrm{w}$ is constant.

It is called the Kaup-Boussinesq system because they have been used Boussinesq scaling in the derivation, and it has been studying by (Kaup, 1975). It has also been used by (Broer, 1974). Also as it goes to the family of long-waves models established by Boussinesq, drawn-out by (Nwogu, 1993; Peregrine, 1967) and many others.

In recent years, the $\mathrm{KB}$ system has been the subject for many other researches. (Zhou, Tian \& Fan, 2009) Work on Solitarywave solution to a dual equation of the $\mathrm{KB}$ system. And (Aminikhah et al., 2016) work on travelling wave solution of nonlinear systems of PDEs by using the factional variable method.

The aim of this paper is solving Kaup-Boussinesq system numerically using ADM and SAM and comparing them with the exact solution. Also the accuracy of the present methods at different values of $x$ and fixed time was discussed.

\section{DESCRIPTION OF THE METHODS}

\subsection{Basic idea of the Adomian decomposition method:}

The principal algorithm of the Adomian decomposition method when applied to a general nonlinear equation has the form. (Adomian, 1986; Ruan \& Lu, 2007). 
$\mathrm{L}_{\mathrm{t}} \mathrm{u}+\mathrm{Ru}+\mathrm{Nu}=\mathrm{g}$

The linear terms are decomposed into $L_{t}+R$, where $L_{t}$ is given to be the operator of the highest order derivatives, $R$ is the reminder of the linear operator and $g$ is the analytic function. While the nonlinear terms are represented by $\mathrm{Nu}$.

Then we get

$\mathrm{L}_{\mathrm{t}} \mathrm{u}=\mathrm{g}-\mathrm{Ru}-\mathrm{Nu}$.

Where $\mathrm{L}_{\mathrm{t}}=\frac{\partial}{\partial \mathrm{t}}$.

Presuming that the inverse of operator $\mathrm{L}_{t}$ exists, it can be written as:

$$
\mathrm{L}_{\mathrm{t}}^{-1}(\cdot)=\int_{0}^{\mathrm{t}}(\cdot) \mathrm{dt}
$$

Applying $\mathrm{L}_{\mathrm{t}}^{-1}$ on the both sides of the equation (2) we get:

$\mathrm{L}_{\mathrm{t}}^{-1} \mathrm{~L}_{\mathrm{t}} \mathrm{u}=\mathrm{L}_{\mathrm{t}}^{-1}(\mathrm{~g})-\mathrm{L}_{\mathrm{t}}^{-1}(\mathrm{Ru})-\mathrm{L}_{\mathrm{t}}^{-1}(\mathrm{Nu})$,

$\mathrm{u}(\mathrm{x}, \mathrm{t})=\mathrm{u}(\mathrm{x}, 0)+\mathrm{L}_{\mathrm{t}}^{-1}(g)-L_{t}^{-1}(\mathrm{Ru})-\mathrm{L}_{\mathrm{t}}^{-1}(\mathrm{Nu})$,

Where $u_{0}=\mathrm{u}(\mathrm{x}, 0)+\mathrm{L}_{\mathrm{t}}^{-1}(\mathrm{~g})$,

Then

$u(x, t)=u_{0}-\mathrm{L}_{\mathrm{t}}^{-1}(\mathrm{Ru})-\mathrm{L}_{\mathrm{t}}^{-1}(\mathrm{Nu})$.

The standard Adomian decomposition method defines the solution $\mathrm{u}(\mathrm{x}, \mathrm{t})$ as the infinite series of the form:

$\mathrm{u}(\mathrm{x}, \mathrm{t})=\sum_{\mathrm{l}=0}^{\infty} \mathrm{u}_{\mathrm{l}}(\mathrm{x}, \mathrm{t})$

Also, $\mathrm{Nu}$ which is usually represented by the sum of series, and it is the nonlinear operator

$\mathrm{Nu}=\sum_{\mathrm{l}=0}^{\infty} \mathrm{A}_{\mathrm{l}}$.

Where $A_{1}$ is Adomian's polynomial of $\left(u_{0}+u_{1}+u_{2}+\right.$ $\cdots+\mathrm{u}_{\mathrm{l}}$ ) which is defined as

$A_{l}=\frac{1}{l !}\left[\frac{d^{1}}{d \lambda^{l}} N\left(\sum_{k=0}^{l} \lambda^{k} u_{k}\right)\right]_{\lambda=0}, l \geq 0$.

\subsection{Basic idea of the Successive Approximation Method:}

One can use this method for solving any initial value problems (Yang et al., 2014)

Let

$\mathrm{u}^{\prime}=\mathrm{f}(\mathrm{t}, \mathrm{u}), \quad \mathrm{u}\left(\mathrm{t}_{0}\right)=\mathrm{u}_{0}$.

It begins by realizing that any solutions to (6) should also be a solution of:

$\mathrm{u}(\mathrm{t})=\mathrm{u}_{0}+\int_{0}^{\mathrm{t}} \mathrm{f}(\mathrm{s}, \mathrm{u}(\mathrm{s})) \mathrm{ds}$.

While we have:

$\mathrm{u}_{\mathrm{M}}(\mathrm{t})=\mathrm{u}_{0}+\int_{0}^{\mathrm{t}} \mathrm{f}\left(\mathrm{s}, \mathrm{u}_{\mathrm{M}-1}(\mathrm{~s})\right) \mathrm{ds}$

A sequence of solution is constructed iteratively to be much closed to the exact solution. And SAM based on the integral as follows:

$$
\mathrm{u}_{0}(\mathrm{t})=\mathrm{u}_{0}
$$

To get $\mathrm{u}_{1}$ we must put initial approximation into equation (7):

$\mathrm{u}_{1}(\mathrm{t})=\mathrm{u}_{0}+\int_{0}^{\mathrm{t}} \mathrm{f}\left(\mathrm{s}, \mathrm{u}_{0}\right) \mathrm{ds}$.

By the same way for $\mathrm{u}_{2}, \mathrm{u}_{3}, \ldots$

\section{NUMERICAL APPLICATIONS:}

The following example is solved numerically by the presented methods:

$$
\begin{array}{r}
u_{t}-v_{x x x}-2(v u)_{x}=0, \\
v_{t}-u_{x}-2 v_{x}=0 .
\end{array}
$$

With the initial conditions: $\mathrm{u}(\mathrm{x}, 0)=\frac{\mathrm{w}^{2}}{2}\left(1+\tanh \left(\frac{\mathrm{wx}}{2}\right)\right)-\frac{w^{2}}{4}\left(1+\tanh \left(\frac{\mathrm{wx}}{2}\right)\right)^{2}$,

And

$\mathrm{v}(\mathrm{x}, 0)=\frac{-\mathrm{w}}{2}\left(1+\tanh \left(\frac{\mathrm{wx}}{2}\right)\right)$.

Where $\mathrm{w}=1.5$, and with soliton solutions (Aminikhah et al., 2016):

$$
\begin{aligned}
\mathrm{u}(\mathrm{x}, \mathrm{t}) & =\frac{\mathrm{w}^{2}}{2}\left(1+\tanh \left(\frac{\mathrm{w}(\mathrm{x}-\mathrm{wt})}{2}\right)\right) \\
& -\frac{\mathrm{w}^{2}}{4}\left(1+\tanh \left(\frac{\mathrm{w}(\mathrm{x}-\mathrm{wt})}{2}\right)\right)^{2},
\end{aligned}
$$

And

$\mathrm{v}(\mathrm{x}, \mathrm{t})=\frac{-\mathrm{w}}{2}\left(1+\tanh \left(\frac{\mathrm{w}(\mathrm{x}-\mathrm{wt})}{2}\right)\right)$,

\subsection{The solution of the nonlinear Kaup-Boussinesq system by ADM:}

By the KB system (1):

$\mathrm{L}_{\mathrm{t}} \mathrm{u}=\mathrm{L}_{\mathrm{xxx}}(\mathrm{v})+2 \mathrm{v}\left(\mathrm{L}_{\mathrm{x}} \mathrm{u}\right)+2 \mathrm{u}\left(\mathrm{L}_{\mathrm{x}} \mathrm{v}\right)$,

$\mathrm{L}_{\mathrm{t}} \mathrm{v}=\mathrm{L}_{\mathrm{x}} \mathrm{u}+2 \mathrm{v}\left(\mathrm{L}_{\mathrm{x}} \mathrm{v}\right)$.

When $\mathrm{L}_{\mathrm{x}}=\frac{\partial}{\partial \mathrm{x}}$, and $\mathrm{L}_{\mathrm{xxx}}=\frac{\partial^{3}}{\partial \mathrm{x}^{3}}$.

$\mathrm{L}_{\mathrm{t}}^{-1}$, which is the inverse operator provided that it exists, is defined as:

$\mathrm{L}_{\mathrm{t}}^{-1}(\cdot)=\int_{0}^{\mathrm{t}}(\cdot) \mathrm{dt}$.

Then apply $\mathrm{L}_{\mathrm{t}}^{-1}$ to (8) and (9) we get:

$$
\begin{aligned}
u(\mathrm{x}, \mathrm{t})= & \mathrm{u}(\mathrm{x}, 0)+\mathrm{L}_{\mathrm{t}}^{-1}\left(\mathrm{~L}_{\mathrm{xxx}} \mathrm{v}\right)+\mathrm{L}_{\mathrm{t}}^{-1}\left(2 \mathrm{vL}_{\mathrm{x}} \mathrm{u}\right) \\
& +\mathrm{L}_{\mathrm{t}}^{-1}\left(2 \mathrm{uL}_{\mathrm{x}} \mathrm{v}\right), \\
\mathrm{v}(\mathrm{x}, \mathrm{t})= & \mathrm{v}(\mathrm{x}, 0)+\mathrm{L}_{\mathrm{t}}^{-1}\left(\mathrm{~L}_{\mathrm{x}} \mathrm{u}\right)+\mathrm{L}_{\mathrm{t}}^{-1}\left(2 \mathrm{vL}_{\mathrm{x}} \mathrm{v}\right),
\end{aligned}
$$

By using initial conditions we get:

$$
\begin{aligned}
\mathrm{u}(\mathrm{x}, \mathrm{t})= & \mathrm{u}_{0}(\mathrm{x})+\mathrm{L}_{\mathrm{t}}^{-1}\left(\mathrm{~L}_{\mathrm{xxx}} \mathrm{v}\right)+\mathrm{L}_{\mathrm{t}}^{-1}\left(2 \mathrm{vL}_{\mathrm{x}} \mathrm{u}\right) \\
& +\mathrm{L}_{\mathrm{t}}^{-1}\left(2 \mathrm{uL} \mathrm{L}_{\mathrm{x}} \mathrm{v}\right), \\
\mathrm{v}(\mathrm{x}, \mathrm{t})= & \mathrm{v}_{0}(\mathrm{x})+\mathrm{L}_{\mathrm{t}}^{-1}\left(\mathrm{~L}_{\mathrm{x}} \mathrm{u}\right)+\mathrm{L}_{\mathrm{t}}^{-1}\left(2 \mathrm{vL}_{\mathrm{x}} \mathrm{v}\right) .
\end{aligned}
$$

Usually the solutions $u(x, t)$ and $v(x, t)$ are defined as an infinite series

$$
\begin{aligned}
\sum_{\mathrm{l}=0}^{\infty} \mathrm{u}(\mathrm{x}, \mathrm{t})= & \mathrm{u}_{0}(\mathrm{x})+\mathrm{L}_{\mathrm{t}}^{-1}\left(\mathrm{~L}_{\mathrm{xxx}} \sum_{\mathrm{l}=0}^{\infty} \mathrm{v}_{\mathrm{l}}\right) \\
& +2 \mathrm{~L}_{\mathrm{t}}^{-1}\left(\sum_{\mathrm{l}=0}^{\infty} \mathrm{A}_{\mathrm{l}}\right)+2 \mathrm{~L}_{\mathrm{t}}^{-1}\left(\sum_{\mathrm{l}=0}^{\infty} \mathrm{B}_{\mathrm{l}}\right), \\
\sum_{\mathrm{l}=0}^{\infty} \mathrm{v}(\mathrm{x}, \mathrm{t})= & \mathrm{v}_{0}(\mathrm{x})+\mathrm{L}_{\mathrm{t}}^{-1}\left(\mathrm{~L}_{\mathrm{x}} \sum_{\mathrm{l}=0}^{\infty} \mathrm{u}_{\mathrm{l}}\right) \\
& +2 \mathrm{~L}_{\mathrm{t}}^{-1}\left(\sum_{\mathrm{l}=0}^{\infty} \mathrm{C}_{\mathrm{l}}\right) .
\end{aligned}
$$

Then find $A_{l}$ which are Adomain polynomials by using equation (5).

While we have

$$
\begin{aligned}
& \mathrm{u}_{0}=\mathrm{u}_{0}(\mathrm{x}), \\
& \mathrm{v}_{0}=\mathrm{v}_{0}(\mathrm{x}) .
\end{aligned}
$$

Then

Where $\mathrm{l} \geq 0$

$$
\begin{aligned}
& \mathrm{u}_{\mathrm{l}+1}=\mathrm{L}_{\mathrm{t}}^{-1}\left(\mathrm{~L}_{\mathrm{xxx}} \mathrm{v}_{\mathrm{l}}+2 \mathrm{~A}_{\mathrm{l}}+2 \mathrm{~B}_{\mathrm{l}}\right), \\
& \mathrm{v}_{\mathrm{l}+1}=\mathrm{L}_{\mathrm{t}}^{-1}\left(\mathrm{~L}_{\mathrm{x}} \mathrm{u}_{\mathrm{l}}+2 \mathrm{C}_{\mathrm{l}}\right) .
\end{aligned}
$$

\subsection{The solution of nonlinear Kaup-Boussinesq system by SAM:}

We apply successive approximation method to approximate solution of the Kaup-Boussinesq system by integrating both sides of the equation (1):

$$
\begin{aligned}
\mathrm{u}(\mathrm{x}, \mathrm{t})= & \mathrm{u}_{0}(\mathrm{x})+\int_{0}^{\mathrm{t}}\left(\frac{\partial^{3} \mathrm{v}(\mathrm{x}, \mathrm{s})}{\partial \mathrm{x}^{3}}+2 \mathrm{v}(\mathrm{x}, \mathrm{s}) \frac{\partial \mathrm{u}(\mathrm{x}, \mathrm{s})}{\partial \mathrm{x}}+\right. \\
& \left.2 \mathrm{u}(\mathrm{x}, \mathrm{s}) \frac{\partial \mathrm{v}(\mathrm{x}, \mathrm{s})}{\partial \mathrm{x}}\right) \mathrm{ds},
\end{aligned}
$$


$\mathrm{v}(\mathrm{x}, \mathrm{t})=\mathrm{v}_{0}(\mathrm{x})+\int_{0}^{\mathrm{t}}\left(\frac{\partial \mathrm{u}(\mathrm{x}, \mathrm{s})}{\partial \mathrm{x}}+2 \mathrm{v}(\mathrm{x}, \mathrm{s}) \frac{\partial \mathrm{v}(\mathrm{x}, \mathrm{s})}{\partial \mathrm{x}}\right) \mathrm{ds}$.

Then the general successive approximation method for the Kaup-Boussinesq system is in the form:

$$
\begin{aligned}
\mathrm{u}_{M}(\mathrm{x}, \mathrm{t})= & \mathrm{u}_{0}(\mathrm{x})+\int_{0}^{\mathrm{t}}\left(\frac{\partial^{3} \mathrm{v}_{\mathrm{M}-1}(\mathrm{x}, \mathrm{s})}{\partial \mathrm{x}^{3}}\right) \mathrm{ds} \\
& +\int_{0}^{\mathrm{t}}\left(2 \mathrm{v}_{\mathrm{M}-1}(\mathrm{x}, \mathrm{s}) \frac{\partial \mathrm{u}_{\mathrm{M}-1}(\mathrm{x}, \mathrm{s})}{\partial \mathrm{x}}\right) \mathrm{ds} \\
& +\int_{0}^{\mathrm{t}}\left(2 \mathrm{u}_{\mathrm{M}-1}(\mathrm{x}, \mathrm{s}) \frac{\partial \mathrm{v}_{\mathrm{M}-1}(\mathrm{x}, \mathrm{s})}{\partial \mathrm{x}}\right) \mathrm{ds}, \\
\mathrm{v}_{\mathrm{M}}(\mathrm{x}, \mathrm{t})= & \mathrm{v}_{0}(\mathrm{x})+\int_{0}^{\mathrm{t}}\left(\frac{\partial \mathrm{u}_{\mathrm{M}-1}(\mathrm{x}, \mathrm{s})}{\partial \mathrm{x}}\right) \mathrm{ds} \\
& +\int_{0}^{\mathrm{t}}\left(2 \mathrm{v}_{\mathrm{M}-1}(\mathrm{x}, \mathrm{s}) \frac{\partial \mathrm{v}_{\mathrm{M}-1}(\mathrm{x}, \mathrm{s})}{\partial \mathrm{x}}\right) \mathrm{ds} .
\end{aligned}
$$

Where $\mathrm{M}=1,2,3, \ldots$

Put $\mathrm{M}=1$ into the equations (10) and (11), we should get $\mathrm{u}_{1}(\mathrm{x}, \mathrm{t})$ and $\mathrm{v}_{1}(\mathrm{x}, \mathrm{t})$ by substituting $\mathrm{u}_{0}(\mathrm{x})$ and $\mathrm{v}_{0}(\mathrm{x})$ which are the initial approximations:

$$
\begin{aligned}
\mathrm{u}_{1}(\mathrm{x}, \mathrm{t})= & \mathrm{u}_{0}(\mathrm{x})+\int_{0}^{\mathrm{t}}\left(\frac{\partial^{3} \mathrm{v}_{0}(\mathrm{x}, \mathrm{s})}{\partial \mathrm{x}^{3}}\right) d s \\
& +\int_{0}^{\mathrm{t}}\left(2 \mathrm{v}_{0}(\mathrm{x}, \mathrm{s}) \frac{\partial \mathrm{u}_{0}(\mathrm{x}, \mathrm{s})}{\partial \mathrm{x}}\right) d \mathrm{~s} \\
& +\int_{0}^{\mathrm{t}}\left(2 \mathrm{u}_{0}(\mathrm{x}, \mathrm{s}) \frac{\partial \mathrm{v}_{0}(\mathrm{x}, \mathrm{s})}{\partial \mathrm{x}}\right) \mathrm{ds} \\
\mathrm{v}_{1}(\mathrm{x}, \mathrm{t})= & \mathrm{v}_{0}(\mathrm{x})+\int_{0}^{\mathrm{t}}\left(\frac{\partial \mathrm{u}_{0}(\mathrm{x}, \mathrm{s})}{\partial \mathrm{x}}\right) \mathrm{ds} \\
& +\int_{0}^{\mathrm{t}}\left(2 \mathrm{v}_{0}(\mathrm{x}, \mathrm{s}) \frac{\partial \mathrm{v}_{0}(\mathrm{x}, \mathrm{s})}{\partial \mathrm{x}}\right) \mathrm{ds} .
\end{aligned}
$$

Put $\mathrm{M}=2$ into the equation (10) and (11) to get $\mathrm{u}_{2}(\mathrm{x}, \mathrm{t})$ and $\mathrm{v}_{2}(\mathrm{x}, \mathrm{t})$

We will do the same steps for $\mathrm{M} \geq 3$.

\subsection{Applying the Adomian Decomposition Method:}

From the example we get:

$$
\left.\begin{array}{rl}
u_{l+1}= & L_{t}^{-1}\left(L_{x x x} v_{l}\right)+L_{t}^{-1}\left(2 A_{l}\right)+L_{t}^{-1}\left(2 B_{l}\right) \\
v_{l+1}=L_{t}^{-1}\left(L_{x} u_{l}\right)+L_{t}^{-1}\left(2 C_{l}\right)
\end{array}\right\}
$$

First for $\mathrm{l}=0$, find $\mathrm{u}_{1}$ and $\mathrm{v}_{1}$

$$
\begin{aligned}
& 2 \mathrm{~A}_{0}= 2\left(\frac{1}{0 !}\left[\frac{\mathrm{d}^{0}}{\mathrm{~d} \lambda^{0}} N\left(\sum_{\mathrm{k}=0}^{0} \lambda^{0} \mathrm{u}_{0}\right)\right]_{\lambda=0}\right), \\
&= 2\left(\mathrm{v}_{0} \frac{\partial u_{0}}{\partial x}\right), \\
&= \frac{\mathrm{w}^{4}}{4} \tanh \left(\frac{\mathrm{wx}}{2}\right) \operatorname{sech}^{2}\left(\frac{\mathrm{wx}}{2}\right) \\
&+\frac{w^{4}}{4} \tanh ^{2}\left(\frac{\mathrm{wx}}{2}\right) \operatorname{sech}^{2}\left(\frac{\mathrm{wx}}{2}\right) . \\
& 2 \mathrm{~B}_{0}= 2\left(\frac{1}{0 !}\left[\frac{\mathrm{d}^{0}}{\mathrm{~d} \lambda^{0}} \mathrm{~N}\left(\sum_{\mathrm{k}=0}^{0} \lambda^{0} \mathrm{u}_{0}\right)\right]_{\lambda=0}\right), \\
&= 2\left(\mathrm{u}_{0} \frac{\partial v_{0}}{\partial x}\right), \\
&= \frac{-\mathrm{w}^{4}}{8} \operatorname{sech}^{2}\left(\frac{\mathrm{wx}}{2}\right)+\frac{w^{4}}{8} \tanh ^{2}\left(\frac{\mathrm{wx}}{2}\right) \operatorname{sech}^{2}\left(\frac{\mathrm{wx}}{2}\right) . \\
& \mathrm{L}_{\mathrm{xxx}}\left(\mathrm{v}_{0}\right)=\frac{-\mathrm{w}^{4}}{4} \tanh ^{2}\left(\frac{\mathrm{wx}}{2}\right) \operatorname{sech}^{2}\left(\frac{\mathrm{wx}}{2}\right)+\frac{\mathrm{w}^{4}}{8} \operatorname{sech}^{4}\left(\frac{\mathrm{wx}}{2}\right) .
\end{aligned}
$$

Then

$$
\begin{aligned}
u_{1}= & \frac{w^{4}}{8} \operatorname{sech}^{4}\left(\frac{w x}{2}\right) t+\frac{w^{4}}{4} \tanh \left(\frac{w x}{2}\right) \operatorname{sech}^{2}\left(\frac{w x}{2}\right) t \\
& -\frac{w^{4}}{8} \operatorname{sech}^{2}\left(\frac{w x}{2}\right) t+\frac{w^{4}}{8} \tanh ^{2}\left(\frac{w x}{2}\right) \operatorname{sech}^{2}\left(\frac{w x}{2}\right) t .
\end{aligned}
$$

Now for $\mathrm{v}_{1}$

$2 \mathrm{C}_{0}=\frac{\mathrm{w}^{3}}{4} \operatorname{sech}^{2}\left(\frac{\mathrm{wx}}{2}\right)+\frac{w^{3}}{4} \tanh \left(\frac{\mathrm{wx}}{2}\right) \operatorname{sech}^{2}\left(\frac{\mathrm{wx}}{2}\right)$.

$\mathrm{L}_{\mathrm{x}}\left(\mathrm{u}_{0}\right)=\frac{-\mathrm{w}^{3}}{4} \tanh \left(\frac{\mathrm{wx}}{2}\right) \operatorname{sech}^{2}\left(\frac{\mathrm{wx}}{2}\right)$

Then

$\mathrm{v}_{1}=\frac{\mathrm{w}^{3}}{4} \operatorname{sech}^{2}\left(\frac{\mathrm{wx}}{2}\right) t$.

By the same way find $u_{2}, v_{2}$ and so on

\subsection{Applying the Successive Approximation Method:}

By applying SAM to the example we get:

$$
\begin{aligned}
\mathrm{u}_{\mathrm{M}}(\mathrm{x}, \mathrm{t})= & \mathrm{u}_{0}(\mathrm{x})+\int_{0}^{\mathrm{t}}\left(\frac{\partial^{3} \mathrm{v}_{\mathrm{M}-1}(\mathrm{x}, \mathrm{s})}{\partial \mathrm{x}^{3}}\right) \mathrm{ds} \\
& +\int_{0}^{\mathrm{t}}\left(2 \mathrm{v}_{\mathrm{M}-1}(\mathrm{x}, \mathrm{s}) \frac{\partial \mathrm{u}_{\mathrm{M}-1}(\mathrm{x}, \mathrm{s})}{\partial \mathrm{x}}\right) \mathrm{ds} \\
& +\int_{0}^{\mathrm{t}}\left(2 \mathrm{u}_{\mathrm{M}-1}(\mathrm{x}, \mathrm{s}) \frac{\partial \mathrm{v}_{\mathrm{M}-1}(\mathrm{x}, \mathrm{s})}{\partial \mathrm{x}}\right) \mathrm{ds}, \\
\mathrm{v}_{\mathrm{M}}(\mathrm{x}, \mathrm{t})= & \mathrm{v}_{0}(\mathrm{x})+\int_{0}^{\mathrm{t}}\left(\frac{\partial \mathrm{u}_{\mathrm{M}-1}(\mathrm{x}, \mathrm{s})}{\partial \mathrm{x}}\right) \mathrm{ds} \\
& +\int_{0}^{\mathrm{t}}\left(2 \mathrm{v}_{\mathrm{M}-1}(\mathrm{x}, \mathrm{s}) \frac{\partial \mathrm{v}_{\mathrm{M}-1}(\mathrm{x}, \mathrm{s})}{\partial \mathrm{x}}\right) \mathrm{ds},
\end{aligned}
$$

When we put $M=1$ into the equations (13) and (14) to obtain the solution of $u_{1}(x, t)$ and $v_{1}(x, t)$ :

$$
\begin{aligned}
\mathrm{u}_{1}(\mathrm{x}, \mathrm{t})= & \mathrm{u}_{0}(\mathrm{x})+\int_{0}^{\mathrm{t}}\left(\frac{\partial^{3} \mathrm{v}_{0}(\mathrm{x}, \mathrm{s})}{\partial \mathrm{x}^{3}}\right) \mathrm{ds} \\
& +\int_{0}^{\mathrm{t}}\left(2 \mathrm{v}_{0}(\mathrm{x}, \mathrm{s}) \frac{\partial \mathrm{u}_{0}(\mathrm{x}, \mathrm{s})}{\partial \mathrm{x}}\right) \mathrm{ds} \\
& +\int_{0}^{\mathrm{t}}\left(2 \mathrm{u}_{0}(\mathrm{x}, \mathrm{s}) \frac{\partial \mathrm{v}_{0}(\mathrm{x}, \mathrm{s})}{\partial \mathrm{x}}\right) \mathrm{ds},
\end{aligned}
$$

Then

$$
\begin{aligned}
\mathrm{u}_{1}= & \frac{\mathrm{w}^{2}}{4}-\frac{w^{2}}{4} \tanh ^{2}\left(\frac{\mathrm{wx}}{2}\right)+\frac{\mathrm{w}^{4}}{8} \operatorname{sech}^{4}\left(\frac{\mathrm{wx}}{2}\right) \mathrm{t} \\
& +\frac{\mathrm{w}^{4}}{4} \tanh \left(\frac{w x}{2}\right) \operatorname{sech}^{2}\left(\frac{w x}{2}\right) \mathrm{t}-\frac{\mathrm{w}^{4}}{8} \operatorname{sech}^{2}\left(\frac{\mathrm{wx}}{2}\right) \mathrm{t} \\
& +\frac{\mathrm{w}^{4}}{8} \tanh ^{2}\left(\frac{\mathrm{wx}}{2}\right) \operatorname{sech}^{2}\left(\frac{\mathrm{wx}}{2}\right) \mathrm{t} .
\end{aligned}
$$

Also

$$
\begin{aligned}
\mathrm{v}_{1}(\mathrm{x}, \mathrm{t})= & \mathrm{v}_{0}(\mathrm{x})+\int_{0}^{\mathrm{t}}\left(\frac{\partial \mathrm{u}_{0}(\mathrm{x}, \mathrm{s})}{\partial \mathrm{x}}\right) \mathrm{ds} \\
& +\int_{0}^{\mathrm{t}}\left(2 \mathrm{v}_{0}(\mathrm{x}, \mathrm{s}) \frac{\partial \mathrm{v}_{0}(\mathrm{x}, \mathrm{s})}{\partial \mathrm{x}}\right) \mathrm{ds},
\end{aligned}
$$

Then

$\mathrm{v}_{1}=\frac{-\mathrm{w}}{2}-\frac{\mathrm{w}}{2} \tanh \left(\frac{\mathrm{wx}}{2}\right)+\frac{\mathrm{w}^{3}}{4} \operatorname{sech}^{2}\left(\frac{\mathrm{wx}}{2}\right) \mathrm{t}$.

By the same way find $\mathrm{u}_{2}, \mathrm{v}_{2}$ and so on 


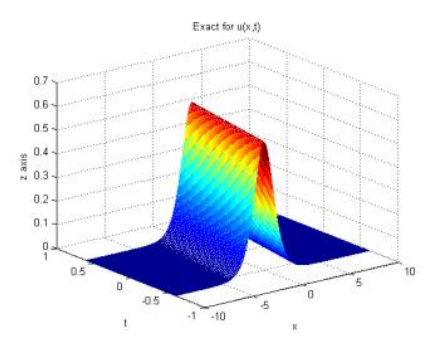

Figure (1): Exact solution for $\mathrm{u}(\mathrm{x}, \mathrm{t})$

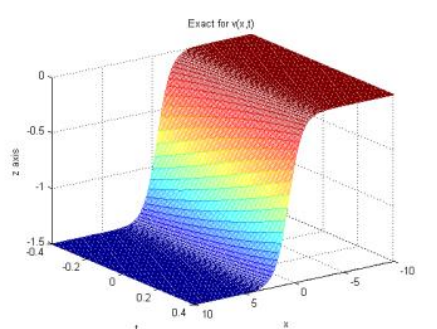

Figure (4): Exact solution for $v(x, t)$

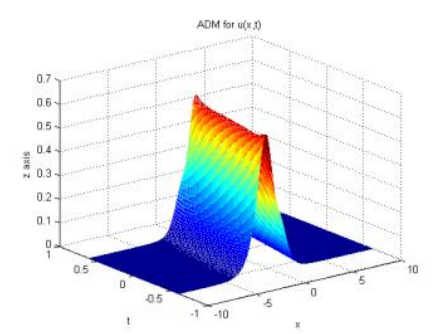

Figure (2): Solution for $\mathrm{u}(\mathrm{x}, \mathrm{t})$ by $\mathrm{ADM}$

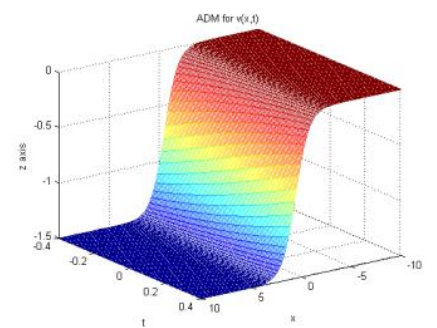

Figure (5): Solution for v(x,t) by ADM

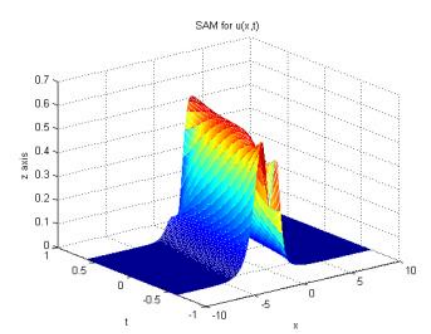

Figure (3): Solution for $\mathrm{u}(\mathrm{x}, \mathrm{t})$ by SAM

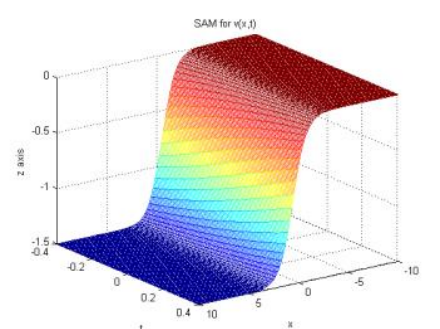

Figure (6): Solution for $v(x, t)$ by SAM

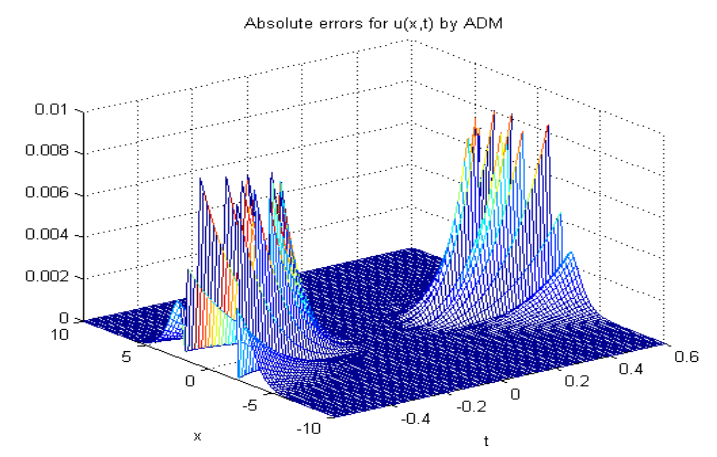

Figure (7): Absolute errors between Exact solution and ADM for $u(x, t)$

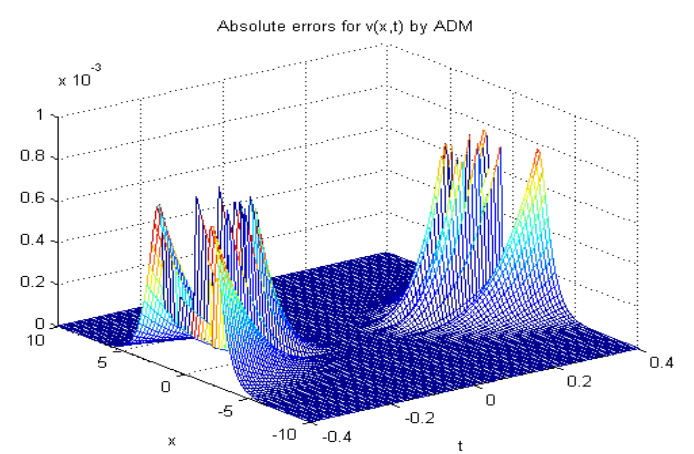

Figure (9): Absolute errors between Exact solution and ADM for $v(x, t)$

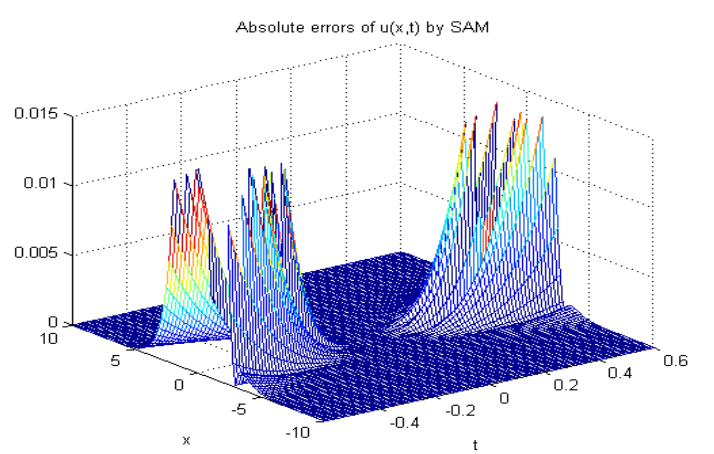

Figure (8): Absolute errors between Exact solution and SAM for $u(x, t)$

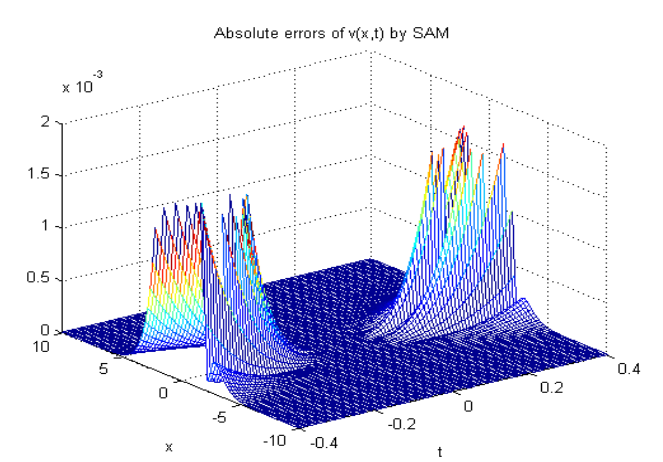

Figure (10): Absolute errors between Exact solution and SAM for $v(x, t)$. 


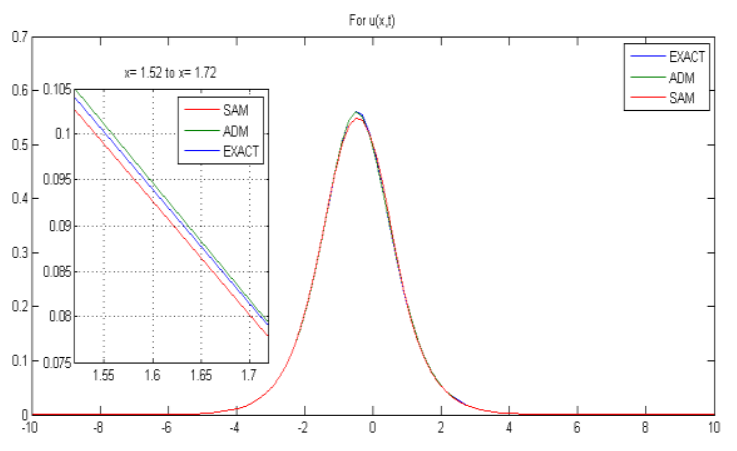

Figure (11): Zooming curves for Exact, ADM and SAM for $u(x, t)$ when $x \in[1.52,1.72], w=1.5$ and $t=-0.3091$

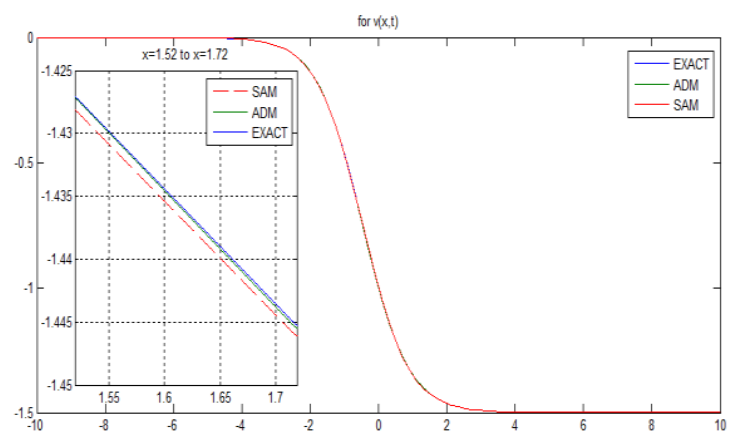

Figure (12): Zooming curves for Exact, ADM and SAM for $v(x, t)$ when $x \in[1.52,1.72], w=1.5$ and $t=-0.3091$

Table (1): Absolute errors between Exact and approximation solutions by ADM and SAM when $x \in[-10,10]$ and $t=-0.3091$ for $u(x, t)$

\begin{tabular}{|c|c|c|c|c|c|c|}
\hline $\mathrm{t}$ & $\mathrm{x}$ & EXACT & $\mathrm{ADM}$ & |EXACT-ADM $\mid$ & SAM & $\mid$ EXACT-SAM $\mid$ \\
\hline \multirow[t]{21}{*}{-0.3091} & -6.16 & 4.36628557E-04 & 4.34180331E-04 & 2.44822561E-06 & 4.34192518E-04 & 2.43603939E-06 \\
\hline & -5.96 & 5.91094413E-04 & 5.87783899E-04 & 3.31051367E-06 & 5.87806227E-04 & 3.28818568E-06 \\
\hline & -5.76 & 8.00166671E-04 & 7.95692213E-04 & 4.47445728E-06 & 7.95733116E-04 & 4.43355479E-06 \\
\hline & -5.56 & 1.08311727E-03 & $1.07707339 \mathrm{E}-03$ & 6.04387469E-06 & $1.07714830 \mathrm{E}-03$ & 5.96896456E-06 \\
\hline & -5.35 & $1.46599271 \mathrm{E}-03$ & 1.45783583E-03 & 8.15688209E-06 & 1.45797297E-03 & 8.01973606E-06 \\
\hline & -5.15 & 1.98397309E-03 & 1.97297708E-03 & $1.09960116 \mathrm{E}-05$ & 1.97322805E-03 & $1.07450396 \mathrm{E}-05$ \\
\hline & -4.95 & 2.68453380E-03 & 2.66973355E-03 & 1.48002533E-05 & 2.67019253E-03 & 1.43412711E-05 \\
\hline & 1.52 & $1.04612682 \mathrm{E}-01$ & 1.05586987E-01 & $9.74305631 \mathrm{E}-04$ & $1.03325496 \mathrm{E}-01$ & 1.28718547E-03 \\
\hline & 1.72 & 7.92777994E-02 & 7.97256092E-02 & 4.47809816E-04 & 7.81453583E-02 & $1.13244106 \mathrm{E}-03$ \\
\hline & 1.92 & 5.96886222E-02 & 5.97929774E-02 & 1.04355137E-04 & 5.87802432E-02 & $9.08379046 \mathrm{E}-04$ \\
\hline & 2.12 & 4.47200203E-02 & 4.46335613E-02 & 8.64589705E-05 & 4.40187357E-02 & 7.01284664E-04 \\
\hline & 2.32 & 3.33823149E-02 & 3.32100205E-02 & $1.72294400 \mathrm{E}-04$ & 3.28495726E-02 & 5.32742250E-04 \\
\hline & 2.53 & 2.48507369E-02 & 2.46555173E-02 & 1.95219591E-04 & 2.44489872E-02 & 4.01749713E-04 \\
\hline & 2.73 & 1.84618344E-02 & 1.82766692E-02 & 1.85165164E-04 & 1.81601193E-02 & $3.01715146 \mathrm{E}-04$ \\
\hline & 5.56 & 2.69727744E-04 & 2.65143718E-04 & 4.58402552E-06 & 2.65117566E-04 & 4.61017785E-06 \\
\hline & 5.76 & 1.99227120E-04 & 1.95835412E-04 & 3.39170788E-06 & 1.95821142E-04 & 3.40597784E-06 \\
\hline & 5.96 & 1.47151324E-04 & 1.44642983E-04 & 2.50834100E-06 & 1.44635198E-04 & 2.51612681E-06 \\
\hline & 6.16 & 1.08686258E-04 & 1.06831853E-04 & $1.85440586 \mathrm{E}-06$ & $1.06827605 \mathrm{E}-04$ & 1.85865363E-06 \\
\hline & 6.36 & 8.02751673E-05 & 7.89045620E-05 & $1.37060524 \mathrm{E}-06$ & 7.89022446E-05 & 1.37292264E-06 \\
\hline & 6.57 & 5.92904754E-05 & 5.82776412E-05 & $1.01283418 \mathrm{E}-06$ & 5.82763770E-05 & $1.01409842 \mathrm{E}-06$ \\
\hline & 6.77 & 4.37911679E-05 & 4.30428191E-05 & 7.48348778E-07 & 4.30421294E-05 & 7.49038455E-07 \\
\hline $\begin{array}{c}\text { Mean } \\
\text { square } \\
\text { error }\end{array}$ & & & & 1.15221469E-06 & & 7.77665407E-06 \\
\hline
\end{tabular}


Table (2): Absolute errors between Exact and approximation solutions by ADM and SAM when $x \in[-10,10]$ at $t=-0.3091$ for $v(x, t)$

\begin{tabular}{|c|c|c|c|c|c|c|}
\hline $\mathrm{t}$ & $\mathrm{x}$ & EXACT & ADM & $\mid$ EXACT-ADM $\mid$ & SAM & |EXACT-SAM| \\
\hline \multirow[t]{21}{*}{-0.3091} & -6.16 & $-2.91142214 \mathrm{E}-04$ & $-2.89507088 \mathrm{E}-04$ & 1.63512641E-06 & $-2.89511152 \mathrm{E}-04$ & 1.63106233E-06 \\
\hline & -5.96 & -3.94166520E-04 & $-3.91954058 \mathrm{E}-04$ & 2.21246246E-06 & $-3.91961505 E-04$ & 2.20501481E-06 \\
\hline & -5.76 & $-5.33634291 E-04$ & $-5.30641328 E-04$ & 2.99296297E-06 & $-5.30654974 \mathrm{E}-04$ & 2.97931644E-06 \\
\hline & -5.56 & $-7.22426110 \mathrm{E}-04$ & -7.18378558E-04 & 4.04755217E-06 & -7.18403558E-04 & 4.02255146E-06 \\
\hline & -5.35 & -9.77966085E-04 & $-9.72494646 \mathrm{E}-04$ & 5.47143877E-06 & -9.72540437E-04 & $5.42564736 \mathrm{E}-06$ \\
\hline & -5.15 & $-1.32381705 E-03$ & $-1.31642502 \mathrm{E}-03$ & 7.39203288E-06 & -1.31650887E-03 & 7.30818694E-06 \\
\hline & -4.95 & -1.79182964E-03 & -1.78185053E-03 & 9.97910579E-06 & -1.78200399E-03 & $9.82564434 \mathrm{E}-06$ \\
\hline & 1.52 & $-1.42667372 E+00$ & $-1.42675189 E+00$ & 7.81779190E-05 & $-1.42778435 E+00$ & 1.11062961E-03 \\
\hline & 1.72 & $-1.44514186 \mathrm{E}+00$ & $-1.44536059 \mathrm{E}+00$ & 2.18738022E-04 & $-1.44600557 \mathrm{E}+00$ & 8.63717647E-04 \\
\hline & 1.92 & $-1.45909194 \mathrm{E}+00$ & $-1.45936352 \mathrm{E}+00$ & 2.71577073E-04 & $-1.45974937 \mathrm{E}+00$ & 6.57430616E-04 \\
\hline & 2.12 & $-1.46956930 \mathrm{E}+00$ & $-1.46984051 \mathrm{E}+00$ & 2.71212054E-04 & $-1.47006472 \mathrm{E}+00$ & 4.95419036E-04 \\
\hline & 2.32 & $-1.47740476 \mathrm{E}+00$ & $-1.47764846 \mathrm{E}+00$ & 2.43696596E-04 & $-1.47777620 \mathrm{E}+00$ & 3.71443070E-04 \\
\hline & 2.53 & $-1.48324570 \mathrm{E}+00$ & $-1.48345151 \mathrm{E}+00$ & $2.05805480 \mathrm{E}-04$ & $-1.48352334 \mathrm{E}+00$ & 2.77631265E-04 \\
\hline & 2.73 & $-1.48758943 \mathrm{E}+00$ & $-1.48775644 \mathrm{E}+00$ & 1.67015105E-04 & $-1.48779646 \mathrm{E}+00$ & $2.07035856 \mathrm{E}-04$ \\
\hline & 5.56 & $-1.49982016 \mathrm{E}+00$ & $-1.49982323 E+00$ & 3.06647441E-06 & $-1.49982324 \mathrm{E}+00$ & 3.07519496E-06 \\
\hline & 5.76 & $-1.49986717 \mathrm{E}+00$ & $-1.49986944 \mathrm{E}+00$ & $2.26684630 \mathrm{E}-06$ & $-1.49986944 \mathrm{E}+00$ & 2.27160420E-06 \\
\hline & 5.96 & $-1.49990189 E+00$ & $-1.49990357 \mathrm{E}+00$ & 1.67534218E-06 & $-1.49990357 \mathrm{E}+00$ & 1.67793795E-06 \\
\hline & 6.16 & $-1.49992754 \mathrm{E}+00$ & $-1.49992878 E+00$ & 1.23797024E-06 & $-1.49992878 \mathrm{E}+00$ & 1.23938636E-06 \\
\hline & 6.36 & $-1.49994648 \mathrm{E}+00$ & $-1.49994740 \mathrm{E}+00$ & 9.14664200E-07 & $-1.49994740 \mathrm{E}+00$ & $9.15436740 \mathrm{E}-07$ \\
\hline & 6.57 & $-1.49996047 \mathrm{E}+00$ & $-1.49996115 E+00$ & 6.75728750E-07 & $-1.49996115 E+00$ & 6.76150200E-07 \\
\hline & 6.77 & $-1.49997081 \mathrm{E}+00$ & $-1.49997130 \mathrm{E}+00$ & 4.99175220E-07 & $-1.49997130 \mathrm{E}+00$ & 4.99405120E-07 \\
\hline $\begin{array}{l}\text { Mean } \\
\text { square } \\
\text { error }\end{array}$ & & & & 2.28397226E-07 & & $1.21403284 \mathrm{E}-06$ \\
\hline
\end{tabular}

\section{CONCLUSION:}

In this paper, successive approximation method (SAM) and Adomian decomposition method (ADM) have been used for solving the kaup-Boussinesq system numerically. In sections three, we give an example to show which method is more accurate and faster than the other, we conclude from the example, that both methods were suitable for solving this kind of problems, they were effective and closed to the exact solution. The results showed that the ADM is more accurate and effective than SAM because it is closer to the exact solution, as shown in table $(1,2)$ and figures $(11,12)$.

\section{REFERENCES}

Adam, B. A. (2015). A comparative study of successive approximation method and he-laplace method. British Journal of Mathematics \& Computer Science, 6(2), 129.

Adomian, G. (1986). A new approaches to the heat equation an application of the decomposition method. Journal of Mathematical Analysis and Applications, 202-209.

Adomian, G. (1986). Nonlinear Stochastic operator equations. San Digo: Academic Press.

Adomian, G. (1994). Solving frontier problems of physics: the decomposition method.

Al Awawdah, E. (2016). The Adomian decomposition method for solving partial differential equations: D.Alaeddin thesis, Birzeit Univercity..

Aminikhan, H., Sheikhani, A. H. R., \& Rezazadeh, H. (2016.). Travelling wave solutions of nonlinear systems of PDEs by using the functional variable method. Boletim da Society Paranaense de Mathematica, 34(2), 213-229.
Biazar, J., \& Ghazvini, H. (2009). Hes homotopy perturbation method for solving systems of volterra integral equations of the second kind. Chaos, Solitons \& Fractals, 39(2), 770-777.

Broer, L. (1974). On the Hamiltonian theory of surface waves. Applied Scientific Research, 29(1), 430-446.

Chen, W., \& Lu, Z (2004). An algorithm for Adomian decomposition method. Applied Mathematics and Computation, 159 (1), 221-235.

Debnath, L. (2011). Nonlinear partial differential equations for scientists and engineers. Springer Science \& Business Media.

Hashem, H. (2015). On successive approximation method for coupled system of Chandrasekhar quadratic integral equations. Journal of the Egyptian Mathematical Society, 23(1), 108-112.

Hosseini, K., Ansari, R., \& Gholamin, P. (2012). Exact solutions of some nonlinear systems of partial differential equations by using the first integral method. Journal of Mathematical Analysis and Applications, 387(2), 807814. doi: 10.1016/j.jmaa.2011.09.044.

Javadi, S. (2014). A modification in successive approximation method for solving nonlinear volttera Hammerstein integral equations of the second kind. Journal of mathematical Extension.

Juliussen, B.-S. H. (2014). Investigation of the Kaup-Boussinesq model equations for water waves: P.Henrik thesis, The University of Bergen.

Kaup, D. (1975). A higher-order water-waves equation and the method for solving it. Progress of Theoretical Physics, 54(2), 396-408.

Koparan, M., Kaplan, M., Bekir, A., \& Guner, O. (2017). A novel generalized Kudryashov method for exact solutions of nonlinear evolution equations. Paper represented at 
thAIP Conference Proceedings, 1798,

doi:10.1063/1.4972674.

Manna, S. A., Easif, F. H., \& Ali, B. Y. (2017). Successive approximation method for solving (1+1)-dimentional dispersive long wave equations. International Journal of Advanced and Applied Science, 4(8), 98-103.

Nwogu, O. (1993). Alternative form of Boussinesq equations for nearshore wave propagation. Journal of waterway, Port, Coastal, and Ocean engineering, 119(6), 618638.

Peregrine, D. H. (1967). Long waves on a beach. Journal of Fluid Mechanics, 27(4), 815-827.

Ruan, J., \& Lu, Z. (2007). A modified algorithm for the adomian decomposition method with applications to lotka-volterra systems. Mathematical and Computer Modeling, 46(9-10), 1214-1224.

Somali, S., \& Gokmen, G. (2007). Adomian decomposition method for nonlinear sturm-liouville problems. Surveys in mathematica and its Applications, 2, 11-20.

Yang, A.-M., Zhang, C., Jafari, H., Cattani, C., \& Jiao, Y. (2014). Picard successive approximation method for solving differential equations arising in fractal heat transfer with local fractional derivative. In Abstract and Applied analysis.

Yassien, S. M. (2014). Modification adomian decomposition method for solving seventh order integro-differential equations.IOSR Journal of Mathematics (IOSR-JM), $72-77$

Zhou, J., Tian, L., \& Fan, X. (2009). Solitary-wave solutions to a dual equations of the Kaup-boussinesq system. Nonlinear Analysis: Real Word Applications 11(4) 3229-3235 